\title{
SUBHARMONIC SOLUTIONS OF SUBQUADRATIC HAMILTONIAN SYSTEMS AND THE MASLOV-TYPE INDEX THEORY
}

\author{
Chong Li \\ Basic Course Department, Beijing Union University, Beijing 100101, P. R. China
}

\begin{abstract}
In this paper, we mainly use the Galerkin approximation method and the iteration inequalities of the Maslov type index theory to study the properties of subharmonic solutions for the Hamiltonian systems $\dot{z}(t)=J \nabla H(t, z(t))$, where $H(t, z)=\frac{1}{2}(\hat{B}(t) z, z)$ $+\hat{H}(t, z), \hat{B}(t)$ is a semipositive symmetric continuous matrix and $\hat{H}$ is unbounded. We prove that these Hamiltonian systems exist subharmonic solutions, and these solutions are pairwise geometrically distinct under certain conditions.
\end{abstract}

Keywords: subharmonic solution, Maslov-type index, Hamiltonian systems.

\footnotetext{
"Corresponding author.

E-mail address: lichong@buu.edu.cn (Chong Li).
}

Copyright ( 2018 Scientific Advances Publishers 2010 Mathematics Subject Classification: 58E05, 34C25, $70 \mathrm{H} 05$.

This work was partially supported by the National Natural Science Foundation of China (11501030), the Support Project of High-level Teachers in Beijing Municipal Universities in the Period of $13^{\text {th }}$ Five-year Plan (CIT\&TCD201704071), and the China Scholarship Council (CSC No. 201708110114).

Submitted by Li Li.

Received August 10, 2018 


\section{Introduction}

In this section, we consider the subharmonic solutions of the Hamiltonian systems

$$
\dot{z}(t)=J \nabla H(t, z(t))
$$

where $J=\left(\begin{array}{cc}0 & -I_{n} \\ I_{n} & 0\end{array}\right)$ is the symplectic matrix, $I_{n}$ is the unit matrix of order $n, H \in C^{2}\left(\mathbb{R} \times \mathbb{R}^{2 n}, \mathbb{R}\right)$ and $\nabla H(t, z)$ is the gradient of $H(t, z)$ respect to $z$. Recall that a solution $z(t)$ of (1.1) is called subharmonic if $z(t)$ is $k T$-periodic for some positive integer $k$. Given an integer $j$ and a $k T$-periodic solution $z$ of (1.1), the phase shift $j * z$ of $z$ is defined by

$$
(j * z)(t)=z(t+j T) .
$$

We shall say two solutions $z_{1}$ and $z_{2}$ are geometrically distinct if

$$
j * z_{1} \neq l * z_{2}, \quad \forall j, l \in \mathbb{Z}
$$

where $\mathbb{Z}$ is the set of all integers.

The generic form of Hamiltonian function $H$ is given by

$$
H(t, z)=\frac{1}{2}(\hat{B}(t) z, z)+\hat{H}(t, z),
$$

where $\hat{B}(t)$ is a $T$-periodic symmetric continuous matrix and $\hat{H}$ has subquadratic behaviour, and $\hat{H}$ and $\hat{B}$ satisfy the following conditions:

(h1) There is a constant $M>0$ such that $|\nabla \hat{H}(t, z)| \leq M$ for all $t \in[0, T]$ and $z \in \mathbb{R}^{2 n}$;

(h2) $\hat{H}(t, z) \rightarrow+\infty$ as $|z| \rightarrow+\infty$ uniformly for $t \in[0, T]$;

(h3) $\hat{B}(t)$ is a $T$-periodic symmetric continuous matrix, $|\hat{B}|_{C^{0}} \leq \delta$ for some $\delta \geq 0$, and $\hat{B}(t)$ is semipositive for all $t \in[0, T]$. 
We state the main result:

Theorem 1.1. Suppose that (h1)-(h3) hold, then for each positive $k,(1.1)$ has a nonconstant $k T$-periodic solution $z_{k}$ such that $z_{j}$ and $z_{p j}$ are geometrically distinct for $p>\left(i_{T}(\hat{B})+\nu_{T}(\hat{B})+n+1\right) /\left(i_{T}(\hat{B})+\nu_{T}(\hat{B})\right.$ $-n+1)$. If all $z_{k}$ are nondegenerate, then $z_{j}$ and $z_{p j}$ are geometrically distinct for $p>1$.

The first result on subharmonic solutions was obtained by Rabinowitz in his pioneer work [17]. Since then, many new contributions have appeared. See, for example, [5, 6, 8, 9, 10, 11, 13] and the references therein. In [6], Ekeland and Hofer proved that under a strict convex condition and a superquadratic condition, the Hamiltonian system (1.1) possesses subharmonic solution $z_{k}$ for each integer $k \geq 1$ and all of these solutions are pairwise geometrically distinct. In [11], Liu generalized the result of [6] to the nonconvex case conditionally. For the subquadratic Hamiltonian systems, many papers, for example, [17] and the references therein, proved the existence of subharmonic solutions.

\section{Preliminaries}

In this section, we briefly recall the Maslov-type index theory for symplectic matrix paths. For the systematic statements of this index theory can be found in reference [13].

As usual, the $2 n$-dimensional symplectic group $S p(2 n)$ is defined by

$$
S p(2 n)=\left\{M \in \mathscr{L}\left(\mathbb{R}^{2 n}\right) \mid M^{T} J M=J\right\},
$$

where $\mathscr{L}\left(\mathbb{R}^{2 n}\right)$ is the set of all real $2 n \times 2 n$ matrices, $M^{T}$ is the transpose of matrix $M$. Denote by $\mathscr{L}_{s}\left(\mathbb{R}^{2 n}\right)$ the subset of $\mathscr{L}\left(\mathbb{R}^{2 n}\right)$ consisting of symmetric matrices. 
In the case of linear Hamiltonian systems

$$
\dot{y}=J B(t) y, \quad \forall y \in \mathbb{R}^{2 n},
$$

where $B \in C\left(\mathbb{R}, \mathscr{L}_{s}\left(\mathbb{R}^{2 n}\right)\right)$ is $T$-periodic. Its fundamental solution $\gamma=\gamma_{B}$ is a symplectic path starting from identity matrix $I_{2 n}$, i.e., $\gamma=\gamma_{B} \in \mathcal{P}(2 n)$ with $\mathcal{P}(2 n)=\left\{\gamma \in C([0, T], S p(2 n)) \mid \gamma(0)=I_{2 n}\right\}$.

In the study of periodic solutions of Hamiltonian systems, an index theory for such symplectic path was introduced by Conley and Zehnder in [4] for nondegenerate elements in $\mathcal{P}(2 n)$ with $n \geq 2$, the $n=1$ case of the nondegenerate elements in $\mathcal{P}(2 n)$ was introduced by Long and Zehnder in [15], the general case for any symplectic path in $\mathcal{P}(2 n)$ was introduced by Long in [14] and Viterbo in [18]. We call this index theory the Maslov-type index theory and denote it by

$$
\left(i_{1}, \nu_{1}\right)=\left(i_{1}(\gamma), \nu_{1}(\gamma)\right) \in \mathbb{Z} \times\{0,1, \cdots, 2 n\},
$$

where $i_{1}=i_{1}(\gamma)$ is the index part or rotation number of $\gamma$ and $\nu_{1}=\nu_{1}(\gamma)$ is the nullity.

The Maslov-type index of $\gamma=\gamma_{B}$ on the interval $[0, T]$ is also called the Maslov-type index of the matrix function $B$. In this case the nullity is defined by

$$
\nu_{T}=\operatorname{dim} \operatorname{ker}\left(\gamma(T)-I_{2 n}\right) .
$$

We know that $B$ is also a $k T$-periodic matrix function, thus its fundamental solution can be extended to the interval $[0, k T]$. In fact, we define a symplectic path $\gamma^{k}:[0, k T] \rightarrow S p(2 n)$ by

$$
\gamma^{k}(t)=\gamma(t-j T) \gamma(T)^{j} \text { for } j T \leq t \leq(j+1) T, \quad 0 \leq j \leq k-1 .
$$


We denote the corresponding Maslov-type index of $\gamma^{k}$ on the interval $[0, k T]$ by

$$
\left(i_{k T}, v_{k T}\right)=\left(i_{k T}(\gamma), \nu_{k T}(\gamma)\right) \equiv\left(i_{k T}\left(\gamma^{k}\right), \nu_{k T}\left(\gamma^{k}\right)\right)
$$

\section{Proof of Theorem 1.1}

Consider the Hamiltonian systems

$$
\left\{\begin{array}{l}
\dot{z}(t)=J \nabla H(t, z(t)), \\
z(0)=z(T) .
\end{array}\right.
$$

Theorem 3.1. Suppose that (h1)-(h3) hold, then (3.1) has a nonconstant T-periodic solution $z$ whose Maslov-type pair satisfies

$$
i_{T}(z) \leq i_{T}(\hat{B})+\nu_{T}(\hat{B})+1 \leq i_{T}(z)+\nu_{T}(z) .
$$

In order to prove Theorem 3.1, we need the following arguments. Let $S_{T}=\mathbb{R} /(T Z)$. Denote $E=W^{1 / 2,2}\left(S_{T}, \mathbb{R}^{2 n}\right)$ the Sobolev space of all $z(t)$ in $L^{2}\left(S_{T}, \mathbb{R}^{2 n}\right)$ whose Fourier series

$$
z(t)=\sum_{k=-\infty}^{k=+\infty} \exp (2 k \pi t J / T) z_{k}, z_{k} \in \mathbb{R}^{2 n}
$$

satisfies

$$
\|z\|_{E}^{2} \equiv T\left|z_{0}\right|^{2}+T \sum_{k=-\infty}^{k=+\infty}|k| \cdot\left|z_{k}\right|^{2}<+\infty .
$$

The inner product of $E$ is defined by

$$
\left(z_{1}, z_{2}\right)_{E}=T\left(z_{0}^{1}, z_{0}^{2}\right)+T \sum_{k=-\infty}^{k=+\infty}|k|\left(z_{k}^{1}, z_{k}^{2}\right)
$$

where $z(t)=\sum_{k=-\infty}^{k=+\infty} \exp (2 k \pi t J / T) z_{k}^{i}, i=1,2$. 
Denote the linear operator $A$ and $\hat{B}$ on $E$ by extending the bilinear form

$$
(A u, v)_{E}=\int_{0}^{T}(-J \dot{u}, v) d t,(\hat{B} u, v)_{E}=\int_{0}^{T}(\hat{B} u, v) d t
$$

Then $A$ is a bounded self-adjoint linear operator and $\hat{B}$ is compact selfadjoint linear operator. Denote the eigenvalues of self-adjoint operator $A-\hat{B}$ by

$$
\cdots \leq \lambda_{2}^{\prime} \leq \lambda_{1}^{\prime}<0<\lambda_{1} \leq \lambda_{2} \leq \cdots .
$$

Let $\left\{e_{j}^{\prime}\right\}$ and $\left\{e_{j}\right\}$ be eigenvectors of $A-\hat{B}$ corresponding to $\lambda_{j}^{\prime}$ and $\lambda_{j}$, respectively. For $m>0$, set

$$
\begin{gathered}
E_{m}^{+}=\operatorname{span}\left\{e_{1}, \cdots, e_{m}\right\}, E_{m}^{-}=\operatorname{span}\left\{e_{1}^{\prime}, \cdots, e_{m}^{\prime}\right\}, \\
E^{0}=\operatorname{ker}(A-\hat{B}), E_{m}=E_{m}^{+} \oplus E_{m}^{-} \oplus E^{0},
\end{gathered}
$$

let $P_{m}: E \rightarrow E_{m}$ be the orthogonal projection. Then $\Gamma=\left\{P_{m}, m=0,1, \cdots\right\}$ is a Galerkin approximation frame with respect to $A-\hat{B}$.

For $d>0$, we denote by $M_{d}^{*}(\cdot), *=+, 0,-$ the eigenspaces corresponding to the eigenvalues $\lambda$ belong to $[d,+\infty),(-d, d)$ and $(-\infty,-d]$, respectively. And denote by $M^{*}(\cdot), *=+, 0,-$ the eigenspaces corresponding to the eigenvalues $\lambda$ belong to $(0,+\infty),\{0\}$ and $(-\infty, 0)$, respectively. For any adjoint operator $Q$, we denote $Q^{\sharp}=\left(\left.Q\right|_{\operatorname{Im} Q}\right)^{-1}$, and we also denote $P^{m} Q P^{m}=\left.\left(P^{m} Q P^{m}\right)\right|_{W_{L}^{m}}$ (see [11]). 
Theorem 3.2. For any continuous T-periodic symmetric matrix function $B(t)$ with the Maslov-type index pair $\left(i_{T}(B), \nu_{T}(B)\right)$, there exists an $m_{0}>0$ such that for $m>m_{0}$,

$$
\begin{aligned}
& \operatorname{dim} M_{d}^{+}\left(P_{m}(A-B) P_{m}\right)=m-i_{T}(B)-\nu_{T}(B)+i_{T}(\hat{B})+\nu_{T}(\hat{B}) \\
& \operatorname{dim} M_{d}^{-}\left(P_{m}(A-B) P_{m}\right)=m-i_{T}(\hat{B})+i_{T}(B) \\
& \operatorname{dim} M_{d}^{0}\left(P_{m}(A-B) P_{m}\right)=v_{T}(B)
\end{aligned}
$$

We also need the following two iteration inequalities (see [11-13]).

Theorem 3.3. For any $k \in \mathbb{N}$, and any continuous T-periodic symmetric matrix function $B(t)$ with the Maslov-type index pair $\left(i_{T}(B), \nu_{T}(B)\right)$, there hold

$$
\begin{gathered}
k\left(i_{T}(B)+\nu_{T}(B)-n\right)+n-\nu_{T}(B) \leq i_{k T}(B) \leq k\left(i_{T}(B)+n\right)-n-\nu_{k T}(B)+\nu_{T}(B), \\
k\left(i_{T}(B)+\nu_{T}(B)-n\right)-n \leq i_{k T}(B) \leq k\left(i_{T}(B)+n\right)+n-\nu_{k T}(B) .
\end{gathered}
$$

Define a function $\hat{\varphi} \in C^{2}(E, \mathbb{R})$ as above, i.e.,

$$
\begin{aligned}
\hat{\varphi}(z) & =\frac{1}{2}(A z, z)_{E}-\int_{0}^{T} H(t, z(t)) d t \\
& =\frac{1}{2}((A-\hat{B}) z, z)_{\mathrm{E}}-\int_{0}^{T} \hat{H}(t, z(t)) d t, \forall z \in E .
\end{aligned}
$$

Let $\hat{\varphi}_{m}=\left.\hat{\varphi}\right|_{E_{m}}$ be the restriction of $\hat{\varphi}$ on $E_{m}$. Similar to [8], we have the following two lemmas.

Lemma 3.1. For every $m>1, \hat{\varphi}_{m}$ satisfies $(P S)_{c}$ condition for every $c \in \mathbb{R}$, i.e., if $\left\{z_{m}\right\} \subset X^{m}$ satisfies $\hat{\varphi}_{m}^{\prime}\left(z_{m}\right) \rightarrow 0$ and $\hat{\varphi}_{m}\left(z_{m}\right) \rightarrow c$, then $\left\{z_{m}\right\}$ has a convergent subsequence. 
Lemma 3.2. $\hat{\varphi}$ satisfies $(P S)_{c}^{*}$ condition for $c \in \mathbb{R}$. i.e., each sequence $\left\{z_{m}\right\}$ such that $z_{m} \in X^{m}, \hat{\varphi}_{m}^{\prime}\left(z_{m}\right) \rightarrow 0$ and $\hat{\varphi}_{m}\left(z_{m}\right) \rightarrow c$ possesses a convergent subsequence in $X$.

In order to prove Theorem 3.1, we need the following definition and the saddle-point theorem (see [7]).

Definition 3.1. Let $E$ be a $C^{2}$-Riemannian manifold and $D$ be a closed subset of $E$. A family of subset of $E, \phi(\alpha)$ is said to be a homological family of dimensional $q$ with boundary $D$ if for some nontrivial class $\alpha \in H_{q}(E, D)$. The family $\phi(\alpha)$ is defined by

$$
\phi(\alpha)=\left\{G \subseteq E: \alpha \text { is in the image of } i_{*}: H_{q}(G, D) \rightarrow H_{q}(E, D)\right\},
$$

where $i_{*}$ is the homomorphism induced by the immersion $i: G \rightarrow E$.

Theorem 3.4. For above $E, D$ and $\alpha$, let $\phi(\alpha)$ be a homological family of dimension $q$ with boundary $D$, suppose that $f \in C^{2}(E, \mathbb{R})$ satisfies (PS) condition. Define

$$
c=\inf _{G \in \phi(\alpha)} \sup _{x \in G} f(x)
$$

Suppose $\sup _{x \in D} f(x)<c$ and $f^{\prime}$ is Fredholm on

$$
\mathscr{K}_{c}(f) \equiv\left\{x \in E: f^{\prime}(x)=0, f(x)=c\right\} .
$$

Then there exists an $x \in \mathscr{K}_{c}(f)$ such that the Morse indices $m^{-}(x)$ and $m^{0}(x)$ of the functional $f$ at $x$ satisfy

$$
m^{-}(x) \leq q \leq m^{-}(x)+m^{0}(x) .
$$


It is clear that a critical point of $\hat{\varphi}$ is a solution of (3.1). For a critical point $z=z(t)$, we define the linearized system at $z$ by $\dot{y}(t)=J H^{\prime \prime}(t, z(t)) y(t)$. Let $B(t)=H^{\prime \prime}(t, z(t))$, then the Maslov-type index pair of $z$ is defined by $\left(i_{T}(z), \nu_{T}(z)\right)=\left(i_{T}(B), \nu_{T}(B)\right)$.

Proof of Theorem 3.1. We also prove this result in 3 steps.

Step 1. The critical points of $\hat{\varphi}_{m}$.

Set $U_{m}=E_{m}^{-} \oplus E^{0}, V_{m}=E_{m}^{+}$. Then $\operatorname{dim} U_{m}=m+\operatorname{dim} E^{0}=m+$ $\nu_{T}(\hat{B}), \operatorname{dim} V_{m}=m$. In the following, we prove that $\hat{\varphi}_{m}(z)$ satisfies:

(1) $\hat{\varphi}_{m}(z) \geq \hat{\beta}_{1}>0, \forall z \in \partial B_{\rho}(0) \cap V_{m}$;

(2) $\hat{\varphi}_{m}(z)<\hat{\xi}_{1}<\hat{\beta}_{1}, \forall z \in \partial Q_{m}$, where $Q_{m}=\left\{r e \mid r \in\left[0, r_{1}\right]\right\} \oplus\left(B_{r_{2}}(0)\right.$ $\left.\cap U_{m}\right), e \in V_{m} \cap \partial B_{1}(0), r_{1}>\rho, r_{2}>0$.

First we prove (1). Take $z \in V_{m}$, we have that $|\hat{H}(t, z)| \leq a+b|z|$ for some constants $a, b$ and all $z \in \mathbb{R}^{2 n}$, then

$$
\begin{aligned}
\hat{\varphi}_{m}(z) & =\frac{1}{2}((A-\hat{B}) z, z)_{E}-\int_{0}^{T} \hat{H}(t, z(t)) d t \\
& \geq \frac{\lambda_{1}}{2}\|z\|_{E}^{2}-\int_{0}^{T} \hat{H}(t, z(t)) d t \\
& \geq \frac{\lambda_{1}}{2}\|z\|_{E}^{2}-\int_{0}^{T}(a+b|z|) d t \\
& \geq \frac{\lambda_{1}}{2}\|z\|_{E}^{2}-\hat{b}_{1}\|z\|_{E}-a T
\end{aligned}
$$

where $\hat{b}_{1}=\hat{a}_{1} b$. Choose $\rho>0$ large enough such that

$$
\frac{\lambda_{1}}{2} \rho^{2}-\hat{b}_{1} \rho-a T \geq \hat{\beta}_{1}>0
$$

which are independent of $m$. 
Now we prove (2). Let $z=z^{0}+z^{-} \in U_{m}$, denote $M_{0}=\|A-\hat{B}\|_{E}$, then

$$
\begin{aligned}
& \hat{\varphi}_{m}(z+r e)=\frac{1}{2}((A-\hat{B})(z+r e), z+r e)_{E}-\int_{0}^{T} \hat{H}(t, z+r e) d t \\
& \quad=\frac{1}{2}\left((A-\hat{B}) z^{-}, z^{-}\right)_{E}+\frac{r^{2}}{2}((A-\hat{B}) e, e)_{E}-\int_{0}^{T} \hat{H}(t, z+r e) d t \\
& \quad \leq \frac{\lambda_{1}^{\prime}}{2}\left\|z^{-}\right\|_{E}^{2}+\frac{M_{0}}{2} r^{2}-\int_{0}^{T} \hat{H}\left(t, z^{0}\right) d t-\int_{0}^{T}\left[\hat{H}(t, z+r e)-\hat{H}\left(t, z^{0}\right)\right] d t,
\end{aligned}
$$

while

$$
\begin{aligned}
\left|\int_{0}^{T} \hat{H}(t, z+r e) d t-\int_{0}^{T} \hat{H}\left(t, z^{0}\right) d t\right| & \leq \int_{0}^{T}\left|\hat{H}(t, z+r e)-\hat{H}\left(t, z^{0}\right)\right| d t \\
& \leq \int_{0}^{T} \int_{0}^{1}\left|\nabla \hat{H}\left(t, z^{0}+s W\right)\right| \cdot|W| d s d t \\
& \leq M \int_{0}^{T}|W| d t \leq \hat{M}_{1} \mid W \|_{E},
\end{aligned}
$$

where $\hat{M}_{1}=\hat{a}_{1} M, W=z^{-}+r e$. Then

$$
\begin{aligned}
\hat{\varphi}_{m}(z+r e) & \leq \frac{\lambda_{1}^{\prime}}{2}\left\|z^{-}\right\|_{E}^{2}+\frac{M_{0}}{2} r^{2}-\int_{0}^{T} \hat{H}\left(t, z^{0}\right) d t+\hat{M}_{1}\|W\|_{E} \\
& =\frac{\lambda_{1}^{\prime}}{2}\left\|z^{-}\right\|_{E}^{2}+\frac{M_{0}}{2} r^{2}-\int_{0}^{T} \hat{H}\left(t, z^{0}\right) d t+\hat{M}_{1}\left\|z^{-}\right\|_{E}+\hat{M}_{1} r .
\end{aligned}
$$

We can choose large enough $r_{1}, r_{2}>\rho$ independent of $\mathrm{m}$ such that

$$
\hat{\varphi}_{m}<\hat{\xi}_{1}<\hat{\beta}_{1}, \forall z \in \partial Q_{m} .
$$


Step 2. Since $Q_{m}$ is deformation retract of $E_{m}$, there holds $H_{q}\left(Q_{m}\right.$, $\left.\partial Q_{m}\right) \cong H_{q}\left(E_{m}, \partial Q_{m}\right)$, where $q=\operatorname{dim} U_{m}+1=m+\nu_{T}(\hat{B})+1=\operatorname{dim} Q_{m}$, and $\partial Q_{m}$ is the boundary of $Q_{m}$ in $U_{m} \oplus\{\mathbb{R} e\}$. But $H_{q}\left(Q_{m}, \partial Q_{m}\right)$ $\cong H_{q-1}\left(S^{q-1}\right) \cong \mathbb{R}$. Denote by $i: Q_{m} \rightarrow P_{m} E$ the inclusion map. Let $\alpha=\left[Q_{m}\right] \in H_{q}\left(Q_{m}, D\right)$ be a generator. Then $i_{*} \alpha$ is nontrivial in $H_{q}\left(P_{m} E, \partial Q_{m}\right)$, and $\phi\left(i_{*} \alpha\right)$ defined by Definition 3.1 is a homological family of dimension $q$ with boundary $D:=\partial Q_{m}$ and $Q_{m} \in \phi\left(i_{*} \alpha\right) . \partial Q_{m}$ and $B_{\rho}(0) \cap V_{m}$ are homologically link. By Lemma 3.1, $\hat{\varphi}_{m}$ satisfies $(P S)_{c}$ condition for $c \in \mathbb{R}$. Define $\hat{c}_{m}=\inf _{G \in \phi\left(i_{*} \alpha\right.} \sup _{z \in G} \hat{\varphi}_{m}(z)$. We have

$$
\sup _{z \in \partial Q_{m}} \hat{\varphi}_{m}(z)<\hat{\xi}_{1}<\hat{\beta}_{1} \leq \hat{c}_{m} \leq \sup _{z \in Q_{m}} \hat{\varphi}_{m}(z) \leq \frac{M_{0}}{2} r_{1}^{2} .
$$

Since $E_{m}$ is finite dimensional, $\hat{\varphi}_{m}^{\prime}$ is Fredholm. By Theorem 3.4, $\hat{\varphi}_{m}$ has a critical point $\hat{z}_{m}$ with critical value $\hat{c}_{m}$, and Morse indices $m^{-}\left(\hat{z}_{m}\right)$ and $m^{0}\left(\hat{z}_{m}\right)$ of $\hat{z}_{m}$ satisfy

$$
m^{-}\left(\hat{z}_{m}\right) \leq m+v_{T}(\hat{B})+1 \leq m^{-}\left(\hat{z}_{m}\right)+m^{0}\left(\hat{z}_{m}\right) .
$$

Since $\left\{\hat{c}_{m}\right\}$ is bounded, passing to a subsequence, suppose $\hat{c}_{m} \rightarrow \hat{c} \in\left[\hat{\beta}_{1}, \frac{M_{0}}{2} r_{1}^{2}\right]$. By $(P S)_{c}^{*}$ condition, passing to a subsequence, there exists an $\hat{z} \in E$ such that

$$
\hat{z}_{m} \rightarrow \hat{z}, \hat{\varphi}(\hat{z})=\hat{c}, \hat{\varphi}^{\prime}(\hat{z})=0 \text {. }
$$

Then $\hat{z}$ is a solution of (3.1). Since $\hat{c}>0, \hat{z}$ is nonconstant. 
Step 3. Let $B(t)=H^{\prime \prime}(t, \hat{z}(t)), d=\frac{1}{4}\left\|(A-B)^{\sharp}\right\|_{E}$. Since

$$
\left\|\hat{\varphi}^{\prime \prime}(x)-(A-B)\right\|_{E} \rightarrow 0 \text { as }\|x-\hat{z}\|_{E} \rightarrow 0
$$

there exists a $r_{3}>0$ such that

$$
\left\|\hat{\varphi}^{\prime \prime}(x)-(A-B)\right\|_{E}<\frac{1}{4} d, \forall x \in V_{r_{3}}(\hat{z})=\left\{x \in E \mid\|x-\hat{z}\|_{E} \leq r_{3}\right\} .
$$

Then for $m$ large enough, there holds

$$
\left\|\hat{\varphi}_{m}^{\prime \prime}(x)-P_{m}(A-B) P_{m}\right\|_{E}<\frac{1}{2} d, \forall x \in V_{r_{3}}(\hat{z}) \cap E_{m}
$$

For $x \in V_{r_{3}}(\hat{z}) \cap E_{m}, \forall u \in M_{d}^{-}\left(P_{m}(A-B) P_{m}\right) \backslash\{0\}$, from above we have

$$
\begin{aligned}
\left(\hat{\varphi}_{m}^{\prime \prime}(x) u, u\right)_{E} & \leq\left(P_{m}(A-B) P_{m} u, u\right)_{E}+\left\|\hat{\varphi}_{m}^{\prime \prime}(x)-P_{m}(A-B) P_{m}\right\|_{E} \cdot\|u\|_{E}^{2} \\
& \leq-\frac{1}{2} d\|u\|_{E}^{2}<0 .
\end{aligned}
$$

Thus

$$
\operatorname{dim} M^{-}\left(\hat{\varphi}_{m}^{\prime \prime}(x)\right) \geq \operatorname{dim} M_{d}^{-}\left(P_{m}(A-B) P_{m}\right), \forall x \in V_{r_{3}}(\hat{z}) \cap E_{m}
$$

Similarly, we have

$$
\operatorname{dim} M^{+}\left(\hat{\varphi}_{m}^{\prime \prime}(x)\right) \geq \operatorname{dim} M_{d}^{+}\left(P_{m}(A-B) P_{m}\right), \forall x \in V_{r_{3}}(\hat{z}) \cap E_{m}
$$

By Theorem 3.2 and above inequalities, for large $m$, we have

$$
\begin{aligned}
m+\nu_{T}(\hat{B})+1 & \geq m^{-}\left(\hat{z}_{m}\right) \\
& \geq \operatorname{dim} M_{d}^{-}\left(P_{m}(A-B) P_{m}\right) \\
& =m-i_{T}(\hat{B})+i_{T}(B) .
\end{aligned}
$$


So we have $i_{T}(\hat{z}) \leq i_{T}(\hat{B})+\nu_{T}(\hat{B})+1$, and we also have

$$
\begin{aligned}
m+\nu_{T}(\hat{B})+1 & \leq m^{-}\left(\hat{z}_{m}\right)+m^{0}\left(\hat{z}_{m}\right) \\
& \leq \operatorname{dim} M_{d}^{-}\left(P_{m}(A-B) P_{m}\right) \oplus \operatorname{dim} M_{d}^{0}\left(P_{m}(A-B) P_{m}\right) \\
& =m+i_{T}(B)+\nu_{T}(B)-i_{T}(\hat{B}) .
\end{aligned}
$$

So we have $i_{T}(\hat{z})+\nu_{T}(\hat{z}) \geq i_{T}(\hat{B})+\nu_{T}(\hat{B})+1$. Combining the above two inequalities, we have

$$
i_{T}(\hat{z}) \leq i_{T}(\hat{B})+\nu_{T}(\hat{B})+1 \leq i_{T}(\hat{z})+\nu_{T}(\hat{z}) .
$$

We also use the following lemma:

Lemma 3.3. Suppose $\hat{B}(t)$ is a T-periodic semipositive symmetric continuous matrix, then $i_{T}(\hat{B})+\nu_{T}(\hat{B}) \geq n$.

Proof of Theorem 1.1. For every positive integer $k, H(t, z)$ is a $k T$-periodic in $t$. By Theorem 3.1, (3.1) has a $k T$-periodic solution $z_{k}$ whose Maslov-type index pair satisfies

$$
i_{T}\left(z_{k}\right) \leq i_{T}(\hat{B})+\nu_{T}(\hat{B})+1 \leq i_{T}\left(z_{k}\right)+\nu_{T}\left(z_{k}\right) .
$$

If $z_{j}$ and $z_{p j}$ are the same geometrically, there exists integer $l$ and $m$ such that

$$
l * z_{j}=m * z_{p j}
$$

where $\left(l * z_{j}\right)(t)=z_{j}(t+l T)$. By Lemma 4.1 of [11],

$$
\begin{gathered}
i_{j T}\left(l * z_{j}\right)=i_{j T}\left(z_{j}\right), v_{j T}\left(l * z_{j}\right)=v_{j T}\left(z_{j}\right), \\
i_{p j T}\left(m * z_{p j}\right)=i_{p j T}\left(z_{p j}\right), v_{p j T}\left(m * z_{p j}\right)=v_{p j T}\left(z_{p j}\right) .
\end{gathered}
$$


Take $k=j, p j$ in (3.2), respectively, we have

$$
i_{p j T}\left(z_{p j}\right) \leq i_{T}(\hat{B})+\nu_{T}(\hat{B})+1 \leq i_{j T}\left(z_{j}\right)+\nu_{T}\left(z_{j}\right) .
$$

Thus by above equations,

$$
\begin{aligned}
i_{T}(\hat{B})+\nu_{T}(\hat{B})+1 & \geq i_{p j T}\left(z_{p j}\right)=i_{p j T}\left(m * z_{p j}\right) \\
& \geq p\left(i_{j T}\left(l * z_{j}\right)+\nu_{T}\left(l * z_{j}\right)-n\right)-n \\
& =p\left(i_{j T}\left(z_{j}\right)+\nu_{j T}\left(z_{j}\right)-n\right)+n \\
& \geq p\left(i_{T}(\hat{B})+\nu_{T}(\hat{B})+1-n\right)+n .
\end{aligned}
$$

Since $\hat{B}(t)$ is semipositive for all $t \in[0, T]$, by Lemma 3.3,

$$
i_{T}(\hat{B})+\nu_{T}(\hat{B})+1-n>0
$$

Hence $p \leq\left(i_{T}(\hat{B})+\nu_{T}(\hat{B})+n+1\right) /\left(i_{T}(\hat{B})+\nu_{T}(\hat{B})-n+1\right)$.

In the case of all $z_{k}$ are nondegenerate, if $z_{j}$ and $z_{p j}$ are the same geometrically, then

$$
\begin{aligned}
i_{T}(\hat{B})+\nu_{T}(\hat{B})+1 & \geq i_{p j T}\left(z_{p j}\right) \\
& \geq p\left(i_{j T}\left(z_{j}\right)+v_{j T}\left(z_{j}\right)-n\right)+n-v_{j T}\left(z_{j}\right) \\
& \geq p\left(i_{T}(\hat{B})+v_{T}(\hat{B})+1-n\right)+n .
\end{aligned}
$$

Then $p \leq 1$.

\section{References}

[1] A. Bahri and H. Berestycki, Forced vibrations of superquadratic Hamiltonian systems, Acta Math. 152(1) (1984), 143-197.

DOI: https://doi.org/10.1007/BF02392196

[2] V. Benci and P. H. Rabinowitz, Critical points theorems for indefinite functionals, Invent. Math. 52(3) (1979), 241-273.

DOI: https://doi.org/10.1007/BF01389883 
[3] K. C. Chang, Infinite Dimensional Morse Theory and Multiple Solution Problems, Birkhäuser, Boston, 1993.

[4] C. Conley and E. Zehnder, Morse-type index theory for flows and periodic solutions for Hamiltonian equations, Comm. Pure Appl. Math. 37(2) (1984), 207-253.

DOI: https://doi.org/10.1002/cpa.3160370204

[5] I. Ekeland, Convexity Method in Hamiltonian Mechanics, Springer-Verlag, Berlin, 1990.

[6] I. Ekeland and H. Hofer, Subharmonics for convex nonautonomous Hamiltonian systems, Comm. Pure Appl. Math. 40(1) (1987), 1-36.

DOI: https://doi.org/10.1002/cpa.3160400102

[7] N. Ghoussoub, Location, multiplicity and Morse indices of min-max critical points, J. Reine Angew. Math. 417 (1991), 27-76.

DOI: https://doi.org/10.1515/crll.1991.417.27

[8] C. Li, The study of subharmonic solutions for subquadratic Hamiltonian systems, Acta Sci. Natur. Uni. Nankai, 47(2014), 59-66.

[9] C. Li, The study of minimal period estimates for brake orbits of autonomous subquadratic Hamiltonian systems, Acta Math. Sinica, English Series 31(10) (2015), 1645-1658.

DOI: https://doi.org/10.1007/s10114-015-4421-3

[10] C. Li, Brake subharmonic solutions of subquadratic Hamiltonian systems, Chin. Ann. Math. 37(3) (2016), 405-418.

DOI: https://doi.org/10.1007/s11401-016-0970-8

[11] C. Liu, Subharmonic solutions of Hamiltonian systems, Nonlinear Anal. TMA 42(2) (2000), 185-198.

DOI: https://doi.org/10.1016/S0362-546X(98)00339-3

[12] C. Liu and Y. Long, Iteration inequalities of the maslov-type index theory with applications, J. Differential Equations 165(2) (2000), 355-376.

DOI: https://doi.org/10.1006/jdeq.2000.3775

[13] Y. Long, Index Theory for Symplectic Paths with Applications, Birkhäuser, Basel, 2000 .

[14] Y. Long, Maslov-type index, degenerate critical points, and asymptotically linear Hamiltonian systems, Science in China (Series A) 33(12) (1990), 1409-1419.

[15] Y. Long and E. Zehnder, Morse Theory for Forced Oscillations of Asymptotically Linear Hamiltonian Systems, In Stoc. Proc. Phys. and Geom. (S. Albeverio et al. Editor) World Sci. (1990), 528-563. 
[16] P. H. Rabinowitz, Minimax Methods in Critical Point Theory with Applications to Differential Equations, CBMS Regional Conf. Ser. in Math., AMS, RI 65 (1986).

[17] P. H. Rabinowitz, On subharmonic solutions of Hamiltonian systems, Comm. Pure Appl. Math. 33(5) (1980), 609-633.

DOI: https://doi.org/10.1002/cpa.3160330504

[18] C. Viterbo, A new obstruction to embedding Lagrangian tori, Invent. Math. 100(1) (1990), 301-320.

DOI: https://doi.org/10.1007/BF01231188 